Pacific Journal of Mathematics

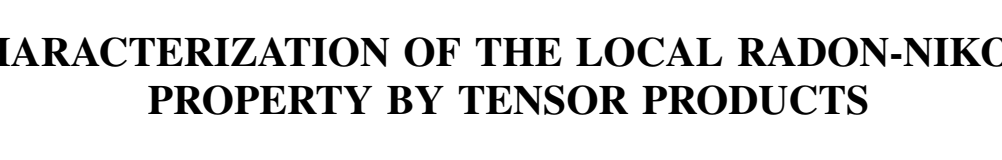




\title{
A CHARACTERIZATION OF THE LOCAL RADON-NIKODYM PROPERTY BY TENSOR PRODUCTS
}

\author{
DONALD P. STORY
}

\begin{abstract}
In this paper, results are presented that characterize the collection of all vector valued measures expressible as an indefinite Bochner integral. More precisely, if $X$ is a Banach space, an $X$-valued vector measure, $\tau$, defined on a measurable space $(S, \Omega)$ is expressible as a Bochner integral if and only if $\tau$ belongs to $c a(S, \Omega) \hat{\otimes}_{\pi} X$, where $\hat{\otimes}_{\pi}$ denotes the strong (or projective) tensor product of two Banach spaces. Other related results are given.
\end{abstract}

Introduction. Throughout this paper, $(S, \Omega)$ will denote a measurable space and $X$ a Banach space. By $c a(\Omega)[\operatorname{cafv}(\Omega ; X)]$ we mean the Banach space of all real valued (resp., $X$-valued) countably additive set functions with finite variation, equipped with the total variation norm $|\cdot|$. Generally, we use the basic notions and notation in Dunford and Schwartz [2].

A vector valued measure $\tau \in \operatorname{cafv}(\Omega: X)$ is said to have the Radon-Nikodym property if whenever $\lambda \in c a(\Omega)$ is a positive measure such that $\tau \ll \lambda$ (that is, $|\tau(E)| \rightarrow 0$ whenever $\lambda(E) \rightarrow 0$ ), then there exists a Bochner integrable function $f: S \rightarrow X$, (see pages 144-154 in [2]) such that

$$
\tau(E)=\int_{E} f d \lambda \text { for all } E \in \Omega .
$$

In this case, $f$ is called the Radon-Nikodym derivative of $\tau$ with respect to $\lambda$. The space of Bochner integrable functions from $S$ into $X$ with respect to a scalar measure $\lambda$ is denoted $B(S, \Omega, \lambda ; X)$; the space of all $X$-valued measures on $\Omega$ that have the $R N$ (RadonNikodym) property is denoted $R N c a(\Omega ; X)$, and forms a closed linear subspace of $\operatorname{cafv}(\Omega ; X)$.

The Main Results. The $R N$ property of a measure is important in classifying certain tensor products of spaces of measures. In preparation for this, we establish an important lemma.

Lemma 1. Suppose $\tau \in \operatorname{cafv}(\Omega ; X)$ such that $\tau \ll \lambda$ and $\lambda \ll \nu$, for some two positive measures $\lambda$ and $\nu$ on $\Omega$. If $\tau$ has a RadonNitodym derivative with respect to $\nu$, then it has a derivative with respect to $\lambda$. 
Proof. By the Lebesgue Decomposition theorem, there exists positive measures $\mu$ and $\sigma$ such that $\nu=\mu+\sigma$ and $\mu \ll \lambda$ and $\sigma \perp \lambda$. Since $\sigma \perp \lambda$, there exists a set $E_{0} \in \Omega$ with $\sigma\left(E_{0}\right)=0$ and $\lambda\left(S-E_{0}\right)=$ 0 . From $\mu \ll \lambda$, there exists an $h \in L_{1}^{+}(S, \Omega, \lambda)$ such that

$$
\mu(E)=\int_{E} h d \lambda \text { for all } E \in \Omega .
$$

Let $f$ denote the derivative of $\tau$ with respect to $\nu$, then for $E \in \Omega$.

$$
\tau(E)=\int_{E} f d \nu=\int_{E} f d \mu+\int_{E} f d \sigma=\int_{E} f h d \lambda+\int_{E} f d \sigma
$$

It is easily seen that $\int_{E} f d \sigma=\int_{E E_{0}} f d \sigma+\int_{E-E_{0}} f d \sigma=0$ for all $E \epsilon$ $\Omega$. Thus, $\tau(E)=\int_{E} f h d \lambda$ and, therefore, $f h$ is the Radon-Nikodym derivative of $\tau$ with respect to $\lambda$.

THEOREM 2. Let $\left\{\tau_{k}\right\} \cong \operatorname{cafv}(\Omega ; X)$ be a sequence of vector measures such that $\sum_{k=1}^{\infty}\left|\tau_{k}\right|(S)<+\infty$. If $\tau_{k}$ has the $R N$ property for each $k$, then so does $\tau=\sum_{k=1}^{\infty} \tau_{k}$.

Proof. Suppose $\lambda \in c a(\Omega)$ is a positive measure such that $\tau \ll \lambda$. Note that $\sum\left|\tau_{k}\right|(E)$ converges absolutely for each $E \in \Omega$, consequently, $\sum\left|\tau_{k}\right|$ defines a $\sigma$-additive measure on $\Omega$ such that $\tau_{n} \ll \sum\left|\tau_{k}\right|$ for each $n$.

Define $\nu=\lambda+\sum\left|\tau_{k}\right|$. Then $\nu$ is a positive measure on $\Omega$ such that $\lambda \ll \nu$; consequently, $\tau \ll \lambda \ll \nu$. It suffices, in view of Lemma 1 to show that $\tau$ has a derivative with respect to $\nu$.

Indeed, for each $n, \tau_{n} \ll \nu$ and $\tau_{n}$ has the $R N$ property implies there exists a function $f_{n} \in B(S, \Omega, \nu ; X)$ such that $\tau_{n}(E)=\int_{E} f_{n} d \nu$. It is easily seen that $\sum_{n=1}^{\infty} f_{n}$ converges in $B(S, \Omega, \nu ; X)$. Therefore, if we define $f=\sum f_{n}$ it is seen that

$$
\tau(E)=\sum \tau_{n}(E)=\sum \int_{E} f_{n} d \nu=\int_{E} \sum f_{n} d \nu=\int_{E} f d \nu .
$$

Thus, $f$ is the derivative of $\tau$ with respect to $\nu$.

We now present the main result of this paper which constitutes a generalization of a theorem of Gil de Lamadrid (Theorem 4.2 [3]). In his paper, he identifies $C^{*}(H) \hat{\boldsymbol{\otimes}}_{\pi} X$ as the class of all regular $X$ valued Radon measures of bounded variation which can be represented as an absolutely convergent series of "step measures." In his paper, $H$ is a compact Hausdorff space, and, of course $C^{*}(H)$ is the space of all regular Radon measures on $H$. 
TheOREm 3. Let $(S, \Omega)$ be a measurable space and $X$ a Banach space, then $c a(\Omega) \hat{\bigotimes}_{\pi} X=R N c a(\Omega ; X)$ isometrically.

Indication of Proof. In [5], we show that $c a(\Omega) \hat{\boldsymbol{\otimes}}_{\pi} X$ can be isometrically embedded in $\operatorname{cafv}(\Omega ; X)$ by the canonical isomorphism

$$
\sum_{i=1}^{k} \mu_{i} \otimes x_{i} \longrightarrow \sum_{i=1}^{k} x_{i} \mu_{i}(\cdot) \text {. }
$$

To prove $c a(\Omega) \hat{\boldsymbol{\otimes}}_{\pi} X=R N c a(\Omega ; X)$, let $\tau \in R N c a(\Omega ; X)$. Put $\lambda=|\tau|$, then $\tau \ll \lambda$. Since $\tau$ has the $R N$ property, there exists a function $f \in B(S, \Omega, \lambda ; X)$ such that $\tau(E)=\int_{E} f d \lambda$ for all $E \in \Omega$.

Because $f$ is Bochner integrable, $f$ can be written in the form $f=\sum_{n=1}^{\infty} x_{n} \xi_{E_{n}} \lambda$ - a.e, where $x_{n} \in X, E_{n} \in \Omega$, and $\sum_{n=1}^{\infty}\left|x_{n}\right| \cdot \lambda\left(E_{n}\right)<+$ $\infty$ (see Brooks [1]). Here $\xi_{E}$ is the characteristic function of the set $E$.

Define $\tau_{n}: \Omega \rightarrow X$ for each positive integer $n$ by $\tau_{n}(E)=x_{n}$. $\lambda\left(E E_{n}\right) . \tau_{n}$ is easily seen to have the $R N$ property and $\tau_{n} \in c \alpha(\Omega) \hat{\boldsymbol{\otimes}}_{\pi} X$. Furthermore,

$$
\sum_{k=1}^{\infty}\left|\tau_{k}\right|(S)=\sum_{k=1}^{\infty}\left|x_{k}\right| \lambda\left(E_{k}\right)<+\infty
$$

Thus, we have

$$
\tau(E)=\int_{E} f d \lambda=\int_{E} \sum x_{k} \xi_{E_{k}} d \lambda=\sum x_{k} \lambda\left(E E_{k}\right),
$$

or,

$$
\tau(E)=\sum_{k=1}^{\infty} \tau_{k}(E) \text { for each } E \in \Omega .
$$

As remarked above $\tau_{k} \in c a(\Omega) \hat{\boldsymbol{\bigotimes}}_{\pi} X$, hence $\sum_{k=1}^{n} \tau_{k} \in c a(\Omega) \hat{\boldsymbol{\bigotimes}}_{\pi} X$ also. Note that (1) implies that the sequence $\left\{\sum_{k=1}^{n} \tau_{k}\right\}$ is Cauchy in $c a(\Omega) \hat{\otimes}_{\pi} X$, because the variation norm is the same as the $\pi$-norm. But by (2), $\sum_{k=1}^{\infty} \tau_{k}$ converges setwise to $\tau$, therefore in variation ( $\pi$-norm). Thus $\tau \in c a(\Omega) \hat{\boldsymbol{\otimes}}_{\pi} X$.

Conversely, if $\tau \in c a(\Omega) \hat{\boldsymbol{\otimes}}_{\pi} X$, by the general theory of projective tensor products (see Trèves [6]), there exists $x_{n} \in X$ and $\lambda_{n} \in$ $c a(\Omega)$ such that $\sum_{k=1}^{\infty}\left|x_{k}\right|\left|\lambda_{k}\right|(S)<+\infty$ and $\tau(E)=\sum_{k=1}^{\infty} x_{k} \lambda_{k}(E)$ for all $E \in \Omega$. Write $\tau_{k}=x_{k} \lambda_{k}$, then clearly $\tau_{k}$ has the $R N$ property, $\tau=\sum \tau_{k}$ and $\sum\left|\tau_{k}\right|<+\infty$. By Theorem 2, $\tau$ has the $R N$ property.

Corollary 1. A measure $\tau \in \operatorname{cafv}(\Omega ; X)$ has the $R N$ property if and only if $\tau$ is expressible as the indefinite Bochner integral with respect to some positive measure. 
Recall that a Banach space $X$ has the Radon-Nikodym property if it's true that any $X$-valued vector measure of finite variation can be expressed as an indefinite Bochner integral.

CoRollary 2. A Banach space $X$ has the Radon-Nikodym property if and only if $c a(S, \Omega) \hat{\boldsymbol{\otimes}}_{\pi} X=c a f v(S, \Omega ; X)$ for every measurable space $(S, \Omega)$.

REMARKs. In particular, if $X$ is reflexive or a separable dual space, then $c a(\Omega) \hat{\boldsymbol{\otimes}}_{\pi} X=\operatorname{cafv}(S, \Omega ; X)$ for every measurable space $(S, \Omega)$. It has been shown that $c a(S, \Omega) \hat{\boldsymbol{\otimes}}_{\pi} X$ is the Banach space, with total variation norm, of all $X$-valued measures on $\Omega$ with the $R N$ property; for sake of completeness, it has been shown in [4] and [5], that $c a(\Omega) \hat{\bigotimes}_{\varepsilon} X$, where $\hat{\bigotimes}_{\varepsilon}$ is the weak (or inductive) tensor product, is the Banach space of all $X$-valued vector measures with relatively norm compact range, equipped with the semi-variation norm. In conclusion, the following question is posed: can the criterion of Corollary 2 be used to give an "external" proof of the fact that reflexive Banach spaces and separable dual spaces have the Radon-Nikodym property?

\section{REFERENCES}

1. J. K. Brooks, Representations of Weak and Strong Integrals in Banach Spaces, Proc. Nat. Acad. Sci., 63 (1969), 266-270.

2. N. Dunford and R. Schwartz, Linear Operators, Part I, Interscience, New York, 1958.

3. J. Gil de Lamadrid, Measures and tensors, Trans. Amer. Math. Soc., 114 (1965), 98-121.

4. D. R. Lewis, Conditional weak compactness in certain inductive tensor products, Math. Ann., 201 (1973), 201-209.

5. D. P. Story, Tensor Products of Spaces of Measures and Vector Integration in Tensor Products Spaces, Dissertation, University of Florida, 1974.

6. F. Trèves, Topological Vector Spaces, Distributions, and Kernels, Academic Press, New York, 1967.

Received May 8, 1979.

University of Akron

Akron, OH 44325 


\section{PACIFIC JOURNAL OF MATHEMATICS}

\section{EDITORS}

DoNALD BABBITT (Managing Editor)

University of Galifornia

Los Angeles, California 90024

HUgo RossI

University of Utah

Salt Lake City, UT 84112

C. C. MOORE AND ANDREW OGG

University of California

Berkeley, CA 94720
J. DugunduI

Department of Mathematics

University of Southern California

Los Angeles, California 90007

R. Finn and J. Milgram

Stanford University

Stanford, California 94305

\section{ASSOCIATE EDITORS}
E. F. BeCKenbaCh
B. H. NeumanN
F. WOLF
K. YoSHIDA

\section{SUPPORTING INSTITUTIONS}

UNIVERSITY OF BRITISH COLUMBIA

CALIFORNIA INSTITUTE OF TECHNOLOGY

UNIVERSITY OF CALIFORNIA

MONTANA STATE UNIVERSITY

UNIVERSITY OF NEVADA, RENO

NEW MEXICO STATE UNIVERSITY

OREGON STATE UNIVERSITY

UNIVERSITY OF OREGON
UNIVERSITY OF SOUTHERN CALIFONIA STANFORD UNIVERSITY UNIVERSITY OF HAWAII UNIVERSITY OF TOKYO UNIVERSITY OF UTAH WASHINGTON STATE UNIVERSITY UNIVERSITY OF WASHINGTON 


\section{Pacific Journal of Mathematics}

\section{Vol. 91, No. $1 \quad$ November, 1980}

Harvey Leslie Abbott, Extremal problems on nonaveraging and nondividing

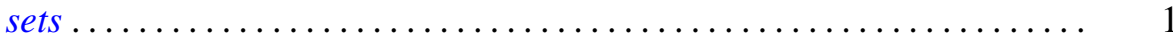

Marine Bruce Abrahamse and Stephen D. Fisher, Mapping intervals to

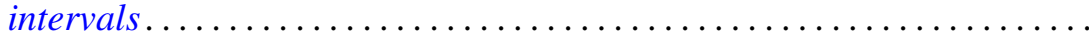

William Wells Adams, The best two-dimensional Diophantine

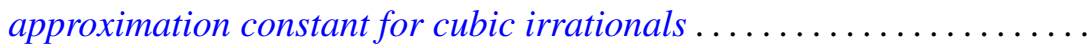

Marilyn Breen, A quantitative version of Krasnosel'skiu 's theorem in

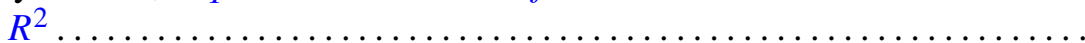

Stephen LaVern Campbell, Linear operators for which $T^{*} T$ and $T T^{*}$

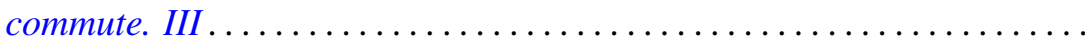

Zvonko Cerin, On cellular decompositions of Hilbert cube manifolds ......

J. R. Choike, Ignacy I. Kotlarski and V. M. Smith, On a characterization

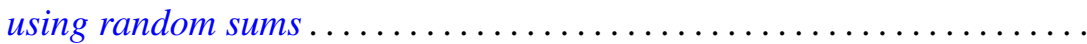

Karl-Theodor Eisele, Direct factorizations of measures .............. 79

Douglas Harris, Every space is a path component space ............. 95

John P. Holmes and Arthur Argyle Sagle, Analytic H-spaces, Campbell-Hausdorff formula, and alternative algebras.............

Richard Howard Hudson and Kenneth S. Williams, Some new residuacity criteria ..........................................

V. Karunakaran and Michael Robert Ziegler, The radius of starlikeness for a class of regular functions defined by an integral ....

Ka-Sing Lau, On the Banach spaces of functions with bounded upper

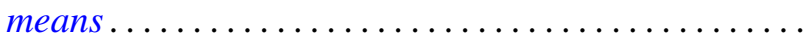

Daniel Paul Maki, On determining regular behavior from the recurrence formula for orthogonal polynomials................

Stephen Joseph McAdam, Asymptotic prime divisors and going down...

Douglas Edward Miller, Borel selectors for separated quotients ..

Kent Morrison, The scheme of finite-dimensional representations of an algebra

Donald P. Story, A characterization of the local Radon-Nikodým property by tensor products

Arne Stray, Two applications of the Schur-Nevanlinna algorithm ...

N. B. Tinberg, The Levi decomposition of a split $(B, N)$-pair ...

Charles Irvin Vinsonhaler and William Jennings Wickless, A theorem on quasi-pure-projective torsion free abelian groups of finite rank... 